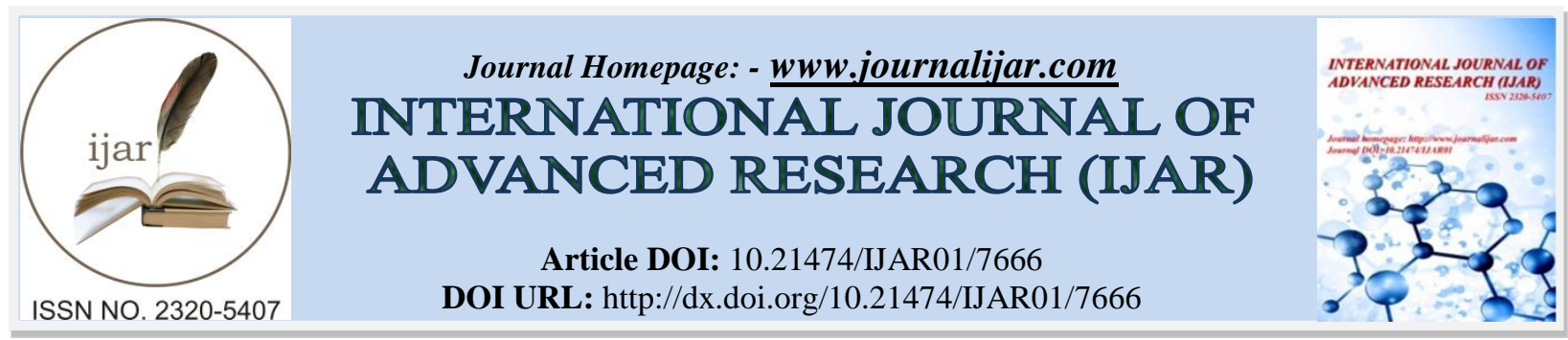

RESEARCH ARTICLE

\title{
COMPARISON OF PRELIMINARY PHYTOCHEMICAL EVALUATION OF FRUIT RIND OF VIBHEETAKI AND BHAVITHA VIBHEETAKI (TERMINALIABELLERICA(GAERTN.ROXB.)
}

Midhun.M ${ }^{1}$ and Dr P Y Ansary ${ }^{2}$.

1. PG Scholar, Dept. of Dravyaguna vijnanam, Govt. Ayurveda College, Tripunithura.

2. Professor \& HOD, Dept. of Dravyaguna vijnanam, Govt. Ayurveda College, Tripunithura.

\section{Manuscript Info}

Manuscript History

Received: 05 July 2018

Final Accepted: 07 August 2018

Published: September 2018

Keywords:-

Terminalia bellerica (Gaertn.)Roxb, BhavithaVibheetaki, Phytochemistry.

\begin{abstract}
Terminalia bellerica(Gaertn.Roxb.) (Combretaceae) is a commonly used Ayurvedic drug as single and in various Ayurvedic formulations. It is explained as Vibheetaki in Ayurvedic classical text books and is an ingredient of Triphala as well. Phytochemical evaluation of a drug helps in its standardization and to justify its use in various formulations. In this study, preliminary physical and phytochemical evaluation of fruit rind of Vibheetaki and Bhavitha Vibheetaki (Terminalia bellerica(Gaertn.Roxb.)including High Performance Thin Layer Chromatography was done. Qualitative analysis showed the presence of alkaloids, tannins, steroid, saponins, proteins and carbohydrates.
\end{abstract}

Copy Right, IJAR, 2018,. All rights reserved.

\section{Introduction:-}

Vibheetaki (Terminalia bellerica(Gaertn.)Roxb) is a drug described extensively in Brhattrayis, Lagutrayis as well as in Nighantus.Vibheetaki is beneficial to cure pathological conditions related to rasa, rakta, mamsa,medo dathus ${ }^{[1]}$.The fruit kalkam of Vibheetaki is a remedy of pathological conditions related to mootra (urine) and asmari(calculus) ${ }^{[2]}$. So the fruits of Vibheetaki is found to be effective in correcting the metabolism of purins, the process of elimination of uric acid through urine ${ }^{[3]}$. Phytochemical analysis of the plant helped in confirming its genuinity. Qualitative evaluation was done to analyze the presence of phyto-constituents. High Performance Thin Layer Chromatography revealed the presence of chemical constituents which help in the identity of the drug. Quantitative evaluation of Vibheetaki and Bhavitha Vibheetaki was done to analyze the increase in potency with Bhavana.

\section{Materia Is and Methods:-}

Materials of Phytochemical analysis:-

Collection of the plants:-

The fruit rind of Vibheetaki (Terminalia bellerica (Gaertn.)Roxb.) was collected from the open market at the Puthiyakavu locality of Thripunithura. The samples were identified as genuine by the Pharmacognostic studies, conducted in the department of Dravyaguna Vijnanam Government Ayurveda College, Thripunithura. It was dried well in sun then powdered and kept in airtight containers. The Bhavitha Vibheetaki was prepared by triturating the powder of fruit rind of Vibheetaki (Terminalia bellerica(Gaertn.(Roxb.) in its own kashaya. This was then subjected to drying and then powdered. The Phytochemical analysis was done at Drug standardization unit of Department of Dravyaguna Vijnanam, Government Ayurveda College, Thripunithura. 


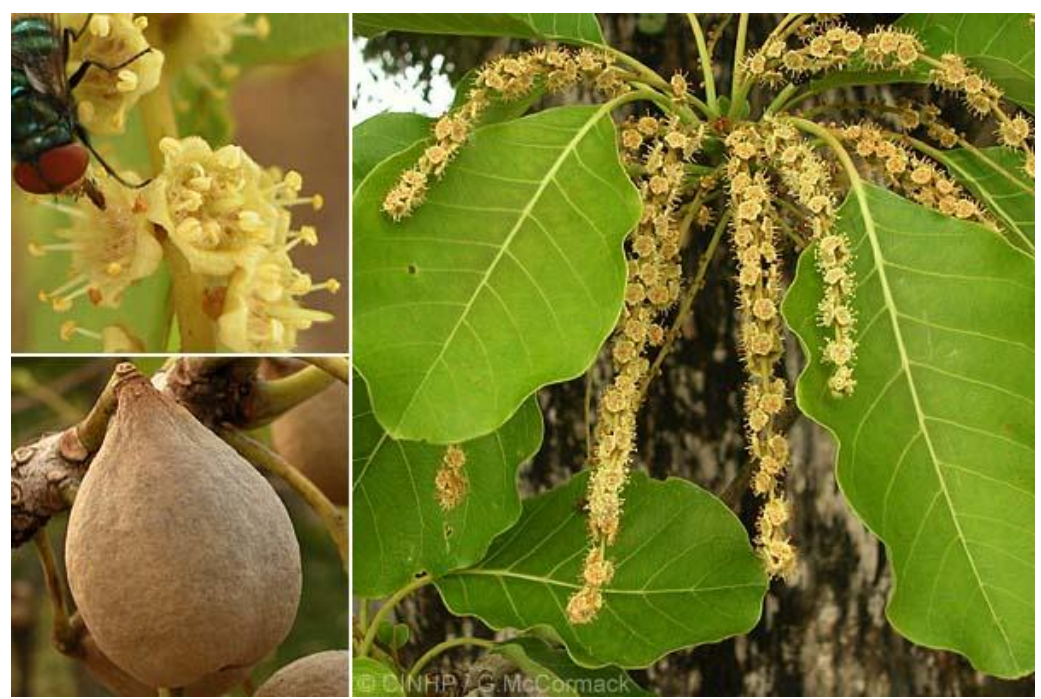

Fig1:-Flower,fruit, inflorescence of Terminalia bellerica

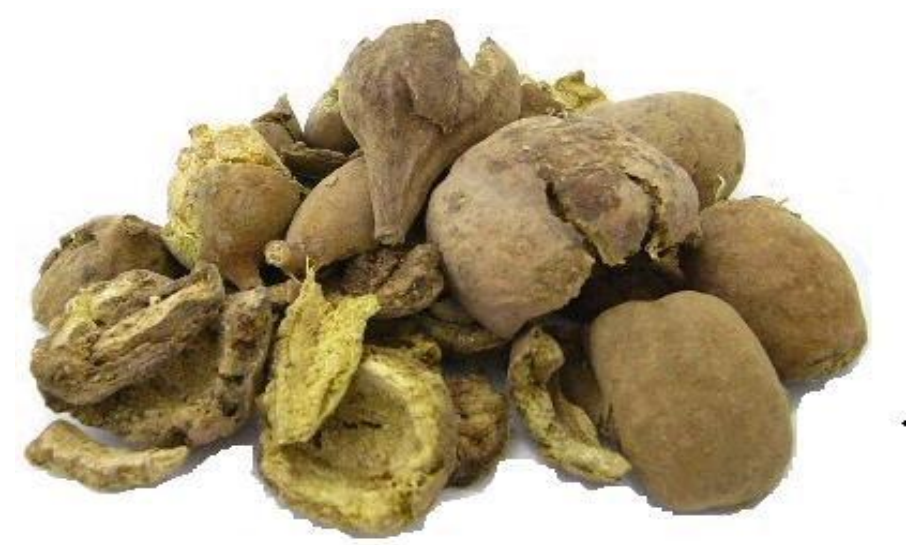

Figure 2:-Fruit rind of Vibheetaki

\section{Process of Bhavana and the need for it:-}

Fruit rind of Vibheetaki collected from the market will be washed thoroughly and dried. Then it is finely powdered to prepare the choorna. Sufficient quantity of kasaya of the fruit rind of Vibheetaki is prepared in the following ratio of boiling $48 \mathrm{~g}$ of thoroughly washed and crushed fruit rinds with 8 times of water. Then it will be reduced to $1 / 8^{\text {th }}$. Bhavana will be done by fully soaking the fine powder in the kasaya, and drying in shade to avoid the loss of essential phytochemicals. After attaining proper dryness, drug will be made into fine powder of mesh size-120. This process will be repeated three times for the preparation of Bhavitha choorna of fruit rind of Vibheetaki. The process of Bhavana aims at the quantitative increase of the essential phytochemicals so as to increase the potency of the drug.

\section{Preliminary Physical and Phytochemical Evaluation:-}

The physical and prelilminary phytochemical analysis was done by standard procedures mentioned in the Ayurvedic Pharmacopoeia of India. Physical evaluation includes Foreign matter, Total ash, Acid insoluble ash, Water insoluble ash, Moisture content, Volatile oil, Fiber content, Tannin content, Total sugar, Reducing sugar, Phenol and pH. Qualitative analysis was done to analyze the presence of steroid, flavonoid, phenol, alkaloid, tannin, carbohydrate, proteins and saponin. Extractive values include water soluble \& alcohol soluble extractives and Successive solvent extraction. HPTLC was done to analyze the presence of chemical constituents in the drug. 
Results:-

Results of physico-chemical parameters:-

Table no 1-Results of physico-chemical parameters

\begin{tabular}{|c|l|c|c|}
\hline SI no & Experiments & Vibheetaki & Bhavitavibheetaki \\
\hline 1 & Foreign matter & $1.2 \%$ & Nil \\
\hline 2 & Total ash & $1.15 \%$ & 1.9 \\
\hline 3 & Acid Insoluble Ash & $2.25 \%$ & 2.4 \\
\hline 4 & Water Insoluble Ash & 19 & - \\
\hline 5 & Moisture Content & Nil & Nil \\
\hline 6 & Volatile oil & $60.6 \%$ & $61.8 \%$ \\
\hline 7 & Fibre & $\mathbf{1 5 . 9 4 \%}$ & $\mathbf{6 3 . 7 5 \%}$ \\
\hline 8 & Tannin Content & $3.17 \%$ & $3.97 \%$ \\
\hline 9 & Total sugar & $2.3 \%$ & $2.5 \%$ \\
\hline 10 & Reducing sugar & $63.5 \mu \mathrm{g}$ & - \\
\hline 11 & Phenol & 4 & 3.5 \\
\hline 12 & $\mathrm{pH}$ & & \\
\hline
\end{tabular}

Results of alcohol and water extractive values:-

Table no 2:-Results of alcohol and water extractive values:

\begin{tabular}{|l|l|c|c|}
\hline SI no & Type of Extractives & Vibheetaki & Bhavitavibheetaki \\
\hline 1 & Cold Alcohol soluble & $8.12 \%$ & $10.9 \%$ \\
\hline 2 & Hot Alcohol soluble & $16.4 \%$ & $16.5 \%$ \\
\hline 3 & Cold water soluble & $36.6 \%$ & $42.68 \%$ \\
\hline 4 & Hot water soluble & $42.68 \%$ & $54 \%$ \\
\hline
\end{tabular}

Results of successive solvent extraction:-

Table no 3:-Results of successive solvent extraction

\begin{tabular}{|c|l|c|}
\hline SI no & Solvents & \% of extractive values of BhavitaVibheetaki \\
\hline 1 & Petroleum ether & $1.2 \%$ \\
\hline 2 & Cyclohexane & $4.04 \%$ \\
\hline 3 & Acetone & $9.24 \%$ \\
\hline & Alcohol & $18.32 \%$ \\
\hline
\end{tabular}

Results of qualitative analysis of crude drugs

Table no 4:-Results of qualitative analysis of crude drugs

\begin{tabular}{|l|l|c|}
\hline \multicolumn{1}{|c|}{ Sl.no } & Experiment & BhavitaVibheetaki \\
\hline 1) & Alkaloids & + \\
a) & Dragendroff's test & + \\
b) & Meyer's test & - \\
\hline 2) & Flavonoids & ++ \\
\hline 3) & Saponins & ++ \\
\hline 4) & Carbohydrates & ++ \\
\hline a) & Fehling's test & + \\
b) & Benedict's test & + \\
\hline 5) & Proteins & + \\
\hline 6) & Phenols & + \\
a) & Ferric chloride test & ++ \\
b) & Lead acetate test & + \\
\hline 7) & Steroids & + \\
\hline 8) & Tannins & \\
a) & Ferric chloride test & \\
b) & Lead acetate test & \\
\hline
\end{tabular}


Results of qualitative analysis of extractives of Bhavitha Vibheetaki:-

Table no 5:-Results of qualitative analysis of extractives of Bhavitha Vibheetaki

\begin{tabular}{|l|l|c|c|c|c|}
\hline Sl no: & Extract & Steroids & Alkaloids & Flavonoids & Phenols \\
\hline 1 & Petroleum ether & + & + & - & - \\
\hline 2 & Cyclohexane & + & ++ & - & - \\
\hline 3 & Acetone & + & - & - & ++ \\
\hline 4 & Alcohol & + & + & - & ++ \\
\hline
\end{tabular}

Results of qualitative analysis of ash of Bhavitha Vibheetaki

Table no 6:-Results of qualitative analysis of ash of Bhavitha Vibheetaki

\begin{tabular}{|c|c|c|}
\hline SI No & Experiment & Bhavitha Vibheetaki \\
\hline \multicolumn{3}{|c|}{ Acid radicals } \\
\hline $\mathbf{1}$ & Carbonate & + \\
\hline 2 & Phosphate & ++ \\
\hline 3 & Chloride & - \\
\hline 4 & Sulphate & + \\
\hline \multicolumn{3}{|c|}{ Basic radicals } \\
\hline 5 & Potassium & - \\
\hline
\end{tabular}

High performance thin layer chromatography (HPTLC):-

High performance thin layer chromatography (HPTLC) was done using the methanolic extract of fruit rind of Vibheetaki (Terminalia bellerica (Gaertn.(Roxb.) having mobile phase as Toluene: Ethyl acetate: Formic acid: Methanol (7:5:1:0:5). The development of the plate is done in the CAMAG 10x10 cm Twin trough chamber and visualized under UV at $254 \mathrm{~nm}$ and $366 \mathrm{~nm}$ after derivatization using 10\% sulphuric acid.

Fig 3:-Tlc Plate Views Of Vibheetaki Phala Churna
AT 254nm
AT 366nm
AT WHITE LIGHT
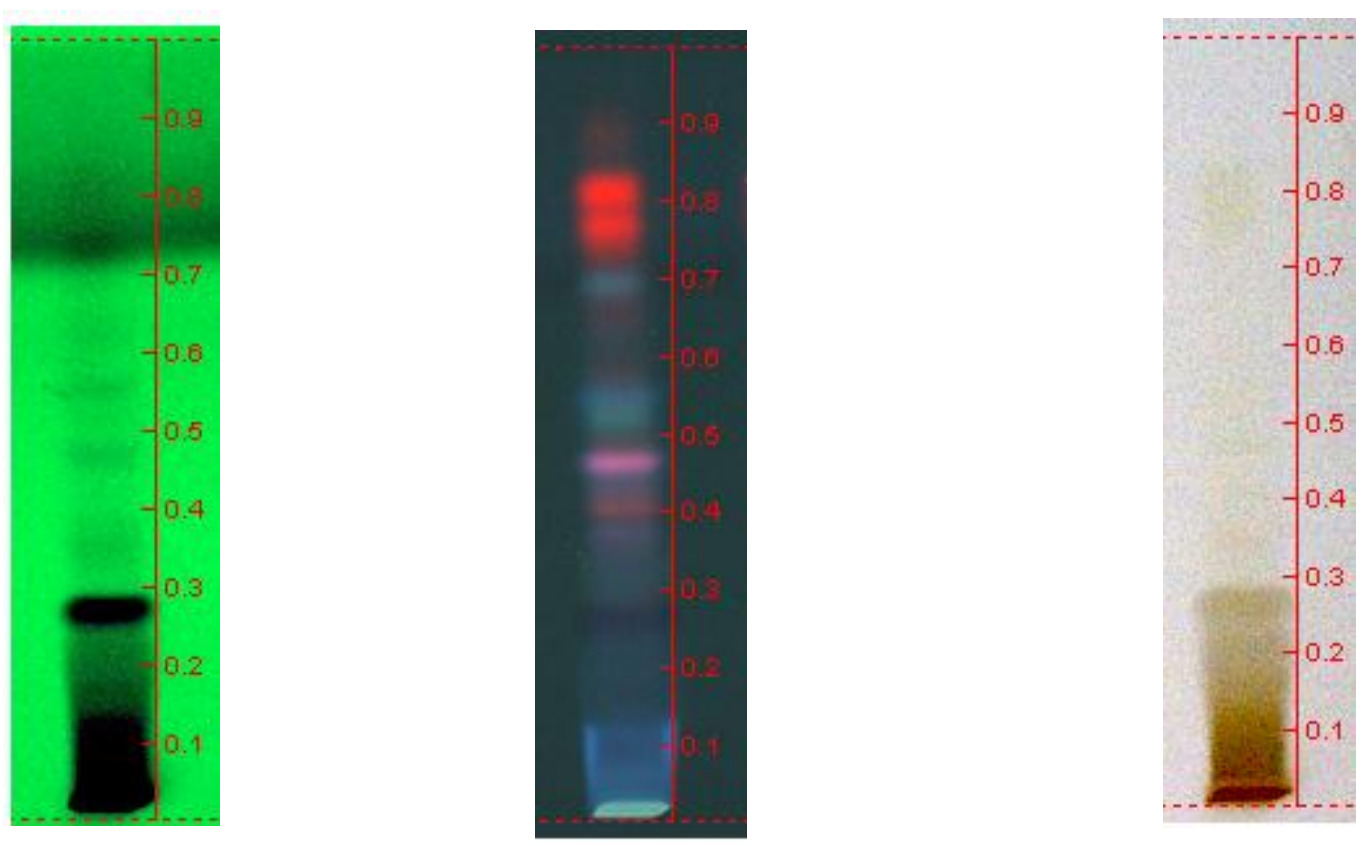
Table No 7:-Rf Value \& \% Area Of Vibheetaki Phala Churnaat 254nm

\begin{tabular}{|c|c|c|c|}
\hline PEAK NO & Rf VALUE & AREA(AU) & \% AREA(AU) \\
\hline 1 & 0.11 & 2107.4 & 3.87 \\
3 & 0.16 & 118.4 & 0.22 \\
4 & 0.26 & 15122.1 & 27.77 \\
6 & 0.34 & 2079.8 & 3.82 \\
7 & 0.47 & 1862.6 & 4.26 \\
8 & 0.55 & 2320.9 & 1.71 \\
\end{tabular}

Total peak no -08

total area $\quad \mathbf{5 4 4 5 2 . 6 ( \mathrm { au } )}$

There is total of 8 peaks with total area of54452.6 A.U at $254 \mathrm{~nm}$. 8 peaks were defined with area2107.4A.U,118.4.U, 15122.1 A.U, 2079.8 A.U, 1862.6A.U, 2320.9A.U, 931 AU,29910.4 A.U respectively. Among them the one major peak were seen at Rf 0.14 and with area $\% 54.93$ A.U.

Table No 8:-Rf Value \& \% Area Of Vibheetaki Phala Churnaat 366nm

\begin{tabular}{|c|c|c|c|}
\hline PEAK NO & Rf VALUE & AREA(AU) & \% AREA(AU) \\
\hline 1 & 0.11 & 2467.2 & 17.29 \\
2 & 0.20 & 460.3 & 10.07 \\
4 & 0.27 & 1437.1 & 3.39 \\
5 & 0.34 & 483.5 & 1.01 \\
6 & 0.37 & 144.3 & 9.36 \\
8 & 0.47 & 1335.8 & 37.93 \\
9 & 0.55 & 5414.0 & 13.92 \\
10 & 0.71 & 1987.1 & 1.56 \\
\hline
\end{tabular}

Total peak no -10

Total area $\quad \mathbf{1 4 2 7 3 . 4}(\mathrm{au})$

There istotal of 10 peaks with total area of14273.4 A.U at $366 \mathrm{~nm} .10$ peaks were defined with area 2467.2A.U,460.3.U, 1437.1 A.U, 483.5 A.U, 144.3 A.U, 1335.8A.U, 5414 A.U, 1987.1 A.U, 223.3 A.U, 320.8 A.U respectively. Among them the one major peak were seen at $\mathrm{Rf} 0.55$ and with area \% $37.93 \mathrm{~A} . \mathrm{U}$

\section{Discussion:-}

The detailed phytochemical analysis was carried out to determine the quality and purity of the drug. Physicochemical parameters such as foreign matter, total ash, acid insoluble ash, water insoluble ash, moisture content, volatile oil content, fibre content, tannin content, phenol content, total sugar and reducing sugar content of the normal drug and bhavitha drug were estimated. . Fibre content, tannin content, total Sugar and reducing sugar content of dried powder of the Vibheetaki and Bhavitha Vibheetakiphala(Terminaliabellerica (Gaertn.) Roxb) were estimated as $60.6 \%$ and $61.8 \% ; 15.94 \%$ and $63.75 \% ; 3.17 \%$ and $3.97 \% ; 2.3 \%$ and $2.5 \%$ respectively.HPTLC analysis of the powder of bhavita Vibheetaki phala (Terminalia bellerica (Gaertn.) Roxb) were also done. In the HPTLC analysis of the drug (Terminalia bellerica (Gaertn.) Roxb) reveals total peaks of 8 with total area of 54452.6 A.U at $254 \mathrm{~nm}$. It also reveals total peaks of 10 with total area of 14273.4 A.U at $366 \mathrm{~nm}$. The tannin content present in the drug Vibheetaki (Terminaliabellerica (Gaertn.) Roxb) may be responsible for its anti hyperuricemic and antiinflammatory properties. From the phytochemical analysis and comparison of normal and bhavitha drug justifies its use in different pathological conditions.

\section{Conclusion:-}

The drug Vibheetaki(Terminaliabellerica (Gaertn.) Roxb) has been widely used in traditional practices as single drug and in different formulations. It is one of the main drug well explained in all Ayurvedic classics. For giving a validation to its therapeutic properties and to standardize the drug the preliminary phytochemical analysis of the 
drug had been carried out. From the phytochemical evaluation of the bhavitha drug, the quantitative increase of its active phytoconstituents was clearly seen. This certainly increase the potency of the drug.

\section{Acknowledgement:-}

I express my immense respect and gratitude towards Dr.P.Y.Ansary, my guide and the Head of the department of Dravyaguna vijnana, Govt. Ayurveda College, Thripunithura, for his immense guidance and motivation throughout the work.

\section{References:-}

1. Caraka Samhitha Sutra sthana, English translation, with CakrapaniDatta's Ayurveda Dipika Commentary, by R.K.Sharma and Bhagwan Dash, Chapter 27, Page:516

2. Susrutha Samhitha Sutra sthana, English translation, by Prof.Sreekanda Murthy, Chapter 46, Page:402

3. Life Extension Magazine- Prevent Gout Attacks by Lowering Uric Acid Levels, andRani.U, Kishan.P, Chandrasekharan.N. A randomized, double blind, placebo controlled, parallel group study to evaluate the effect of Terminaliachebula, Terminaliabellericaand Febuxostat in patients with hyper urecemia

4. Bhaishajyaratnavali, English translation, Vol-1, Chapter-4, Sloka:119, Page:90 